\title{
XLVIII. Electrochemical effects due to magnetization
}

\section{George Owen Squier Ph.D. Lieut. U.S. Army}

To cite this article: George Owen Squier Ph.D. Lieut. U.S. Army (1893) XLVIII. Electrochemical effects due to magnetization, Philosophical Magazine Series 5, 35:217, 473-489, DOI:

$10.1080 / 14786449308621419$

To link to this article: http://dx.doi.org/10.1080/14786449308621419

曲 Published online: 08 May 2009.

Submit your article to this journal $\lceil\pi$

Џ Article views: 3

Q View related articles $₫$ 
LONDON, EDINBURGH, AND DUBLIN

\title{
PHILOSOPHICAL MAGAZINE
}

\author{
AND \\ JOURNAL OF SCIENCE.
}

[FIFTH SERIES.]

$J U N E 1893$.

XLVIII. Electrochemical Effects due to Magnetization. By Georgr OWen Squier, Ph.D., Lieut. U.S. Army *.

\section{INTRODUCTION.}

THE influence of magnetism on chemical action was the 1 subject of experiment by numerous investigators during he first half of the present century $\dagger$. Up to 1847 we find by no means a uniformity of statement in regard to this subject, and secondary effects were often interpreted as a true chemical influence. Among the earlier writers who maintained that such an influence exists may be mentioned Ritter, Schweigger, Döbereiner, Fresnel, and Ampère; while those of opposite view were Wartmann, Otto-Linné Erdmann, Berzelius, Robert Hunt, and the Chevalier Nobili.

Professor Remsen's discovery, in 1881, of the remarkable influence of magnetism on the deposition of copper from one of its solutions on an iron plate, again attracted attention to the subject, and since then considerable work has been done directly or indirectly bearing on the question.

Among other experiments by Professor Remsen $\ddagger$ were the action in the magnetic field of copper on zinc, silver on zinc, copper on tin, and silver on iron, in all of which cases the magnet evidently exerted some influence. With copper sulphate on an iron plate the effects were best exhibited, the

* Communicated by the Author.

† Wartmann, Philosophical Magazine, 1847, (3) xxx. p. 264.

$\ddagger$ American Chemical Journal, vol. iii. p. 157, vol. vi. p. 430 ; 'Science,' vol. i. no. 2 (1883).

Phil. Mag. S. 5. Vol. 35. No. 217. June 1893. 2 L 
copper being deposited in lines approximating to the equipotential lines of the magnet, and the outlines of the pole being distinctly marked by the absence of deposit.

Messrs. Nichols and Franklin * were the next to conduct experiments bearing on this subject. They found that finely divided iron which has become "passive "through the action of strong nitric acid suddenly regains its activity when introduced in a magnetic field, and also that when one of the two electrodes immersed in any liquid capable of chemically acting upon them is placed in a magnetic field, a new difference of potential is developed between them due to this magnetization. They ascribe these effects to electric currents in the liquid produced indirectly by the magnet, which currents go in the liquid from the magnetized to the neutral electrode.

Professor Rowland and Dr. Louis Bell † were the first to note the "protective action" of points and ends of magnetic electrodes, and to give the exact mathematical theory of this action. Their results were directly opposite to those of Messrs. Nichols and Franklin, who found, as stated above, that points and ends of bars in a magnetic field acted like zincs to the other portions, or were more easily dissolved by the liquid.

The method of experiment adopted by Professor Rowland was to expose portions of bars of the magnetic metals placed in a magnetic field to reagents which would act upon them chemically, and study the changes in the electro-chemical nature of the exposed parts by fluctuations in a delicate galvanometer connected with the two bars. Iron, nickel, and cobalt were experimented upon, and nearly thirty reagents were examined in this manner. The results are summed up in the following statement:- "When the magnetic metals are exposed to chemical action in a magnetic field, such action is decreased or arrested at any points where the rate of variation of the square of the magnetic force tends towards a maximum."

Other investigations in this field are those of Andrews who employed iron and steel bars from eight to ten inches long with their ends immersed in various solutions, and one bar magnetized by means of a solenoid. The protective action was not noted, but, on the contrary, the magnetized

* American Journul of Scienee, vol. xxxi. p. 27.2, vol. xxxiv. p. 419, vol. xxxv. p. 290.

f Phil. Mag. vol. xxvi. p. 105.

I Proceedings of the Royal Society, no. 44, pp. 152-168, and no. 46, pp. $176-193$. 
bars acted as zines to the neutral bars, thus indicating that they were more easily attasked.

Practically the same results were obtained by Dr. Theodor Gross *; soft iron wires, $8 \mathrm{~cm}$. long and $3 \mathrm{~cm}$. in diameter, coated with sealing-wax except at the ends were exposed to various liquids. When one electrode was magnetized, a current was obtained going in the liquid from the magnetized electrode to the non-magnetized electrode.

It thus appears that there is at least an apparent inconsistency between the protective results of Professor Rowland and Professor Remsen, and those of Nichols, Andrews, Gross, and others, who find the more strongly magnetized parts of iron electrodes more easily attacked than the neutral parts; and it was with the object of endeavouring to reconcile these results, and of studying the exact nature of the influence exerted by the magnet, that the experiments recorded in this paper were undertaken.

\section{Apparatus and Method of Investigation.}

The method of investigation was that adopted by Professor Rowland in his previous work on the subject, since its facility and delicacy permitted the effects of the magnet to be observed whenever there was the slightest action on the electrodes by the solution examined, and the investigation could thus be carried over a wide range of material.

A large electromagnet was employed to furnish the magnetic field, and, at a distance sufficient to prevent any direct influence due to the magnet, a delicate galvanometer of the Rowland type was set up. Small cells were made with iron electrodes of special forms, coated with sealing-wax except at certain parts, and immersed in a liquid capable of acting chemically on iron. The whole was contained in a 50 cubic centim. glass beaker, and when joined to the connecting wires of the distant galvanometer was firmly clamped between the poles of the electromagnet.

In the course of the examination of a number of substances it was found necessary to use two galvanometers-one specially made by the University instrument-maker and very sensitive, which was employed with acids which evolve hydrogen; the other, much less sensitive, was best suited to the violent "throws" with nitric acid and iron. The samples of iron used throughout the experiments were obtained from

* "Ueber eine neue Entstehungsweise galvanischer Strome durch Magnetismus," Sitzungsberichte der Wiener Akademie, 1885, vol. xcii. (1885) p. 1373. 
Carnegie, Phipps, and Co., of Pittsburg, and were practically pure.

In order to insure a uniform density of surface, the electrodes were turned from the same piece and polished equally with fine emery-cloth. The magnet could be made or reversed at the galvanometer, and its strength varied at will by a non-inductive resistance. The electrochemical effects due to the magnetic field could thus be studied with facility by the fluctuations of the galvanometer-needle. The original difference of potential, which always existed between the electrodes, was compensated by a fraction of a Daniell cell, so the effects of a variation of the magnetic field could be observed when no original current was passing between the electrodes.

The standard cells were made with care, and ander uniform treatment possessed at $20^{\circ} \mathrm{C}$. an electromotive force of 1.105 volt. The connexions with the compensating circuit, which contained a finely-divided bridge, were so arranged that from its readings the difference of potential between the distant electrodes became known at once without involving the resistance of the cell or of the galvanometer.

Since quantitative measurements of the effects observed were desired, a preliminary step was to calibrate the electromagnet for a given distance apart of the pole-pieces. The method employed was the well-known one of comparing the galvanometer deflexions produced by a test-coil in the field with those of an earth inductor in series in the circuit. Since the effect of the sudden addition of a certain strength of field was wanted instead of its absolute value, the deflexions with the test-coil were taken for simple "make" or "break" and not for reversed field, thus eliminating the residual magnetism of the pole-pieces.

In the formula applicable, viz.,

$$
\frac{\mathrm{H}}{\mathrm{H}^{\prime}} \frac{\pi n a^{2}}{\pi n^{\prime} a^{\prime 2}}=\frac{d}{d^{\prime}} \text { nearly, }
$$

in which $d$ and $d^{\prime}$ represent the deflexions due to the inductor and test-coil respectively, $\mathrm{H}$ and $\mathrm{H}^{\prime}$ the carth's field and the field to be measured, $n$ and $n$ the number of turns, and $a$ and $a^{\prime}$ the radii of the coils, the particular values were :-

$$
\begin{aligned}
\pi n a^{2} & =20716 \text { square centim. } \\
\pi n^{\prime} a^{22} & =6 \cdot 788, \text { square centim. } \\
d & =4 \cdot 697 .
\end{aligned}
$$

Distance between pole-pieces 3.5 centim. 
$\mathrm{H}^{\prime}=1299^{\circ} 48 d^{\prime} \mathrm{H}$, and as $d^{\prime}$ varied from $\frac{1}{20}$ to 16 , the range of field employed was from 65 to $20,800 \mathrm{H}$.

A curve was constructed so that from accurate ammeter readings in the field circuit the strength in absolute measure could be read off at once.

\section{EXPerLmental Results.}

Preliminary.-The first experiments were made with very dilute nitric acid and iron electrodes-one a circular disk of 5 millim. radius, and the other a small wire 1 centim. long and 1 millim. in diameter, turned to a sharp point at one end. The point was placed opposite the centre of the disk, at a distance of 1 centim. from it, and the whole placed so that the cylindrical electrode coincided with the direction of the lines of force. When the minute point and the centre of the disk were exposed to the liquid, and the magnet excited, a momentary "throw" of the galvanometer was observed in the direction indicating the point as being protected or acting as the copper of the cell.

When the pointed pole was slightly flattened at the end, and the insulation so cut away that the surfaces of exposure on the two electrodes were exactly the same, the throw of the galvanometer on making the field was very much diminished, although still perceptible, since the disposition of lines of force would still be very different over the two plane surfaces of exposure.

With ball-and-point electrodes precisely similar phenomena were observed as with a disk and point, except to a less degree.

The gradual reversal of the current shortly after exciting the field, the independence of the throw of the direction of the current through the magnet, the disappearance of the throw when the nature of the magnetic field at the exposed parts became the same, and the effects of artificially stirring the liquid, were observed exactly as described by Messrs. Rowland and Bell.

In the course of a large number of preliminary experiments with nitric acid, it was soon observed that under certain conditions the effect of suddenly putting on the magnetic field was to produce a less rapid deflexion of the galvanometer in the opposite direction, or indicating the point as acting as a zinc. Plainly this irregular behaviour, due to the magnet, required a more systematic study than it had yet received. It had been found that the reversal of the current, which regularly followed the "protective throw," was decreased or destroyed by anything which prevented free 
circulation in the liquid, and that an acidulated gelatine, which was allowed to harden around the poles, was best suited for this purpose. The great irregularity observed in any one experiment made it necessary to eliminate everything possible which might mask the true phenomenon, if any accurate comparisons were to be drawn between the effects observed in the different cases; accurdingly a standard form of experiment was adopted, which was carefully repeated many times. The cell found best suited for this purpose was composed as follows :-

\begin{tabular}{|c|c|}
\hline Disk electrode, diameter. & $14 \cdot 4$ millim. \\
\hline " " thickness. & $2 \cdot 6$ \\
\hline Point electrode, total length & $15 \cdot 2$ \\
\hline $\begin{array}{l}\text { diameter } \ldots \ldots \ldots \\
\text { length of point. }\end{array}$ & $\begin{array}{l}4 \cdot 4 \\
5 \cdot 2\end{array}$ \\
\hline Distance of point from centre of & 10 \\
\hline
\end{tabular}

The same electrodes were used throughout any set of experiments, being carefully cleaned and polished each time.

With nitric acid the liquid was finally made up as follows :-

Distilled water 10 grammes.

Hard gelatine 1 gramme.

C. P. nitric acid (sp. gravity 1.415) 0.533 gramme.

The gelatine and water were allowed to stand until the former had dissolved without the application of heat, when the acid was added and the whole thoroughly mixed. Too strongly acidulated gelatine would not harden at all.

In some cases, in order to protect the point from the beginning, the electrodes, secured as usual at the ends of two small glass tubes containing the connecting wires, were tirmly clamped in the proper position between the poles of the magnet, and the magnetic field put on before the cell was completed, by pushing the beaker containing the solution up in position round the electrodes.

With this cell a series of parallel experiments were conducted to obtain the variation of the effects with time, the amount of iron salts present, the fluidity of the solution, and with constant and variable magnetic fields.

\section{A. Behaviour of the Cell with Time, in the Earth's Field.}

The cell was placed entirely outside the magnetic field, and galvanometer-readings taken at intervals of one minute for three hours. The curve fig. 1 (I.) shows these results. Positive ordinates indicate a current from the point to the disk, 
and negative ordinates the reverse current. Other experiments with fresh solutions, same electrodes, same exposed area, and every condition as nearly as possible the same, gave curves of practically the same character, and the one given is selected to illustrate.

The curve indicates that the original current was to the point electrode; this gradually decreased, owing to polarization, until after a hour and five minutes it reversed slightly, but again reversed thirty-five minutes later, and after a littlo more than two hours the deflexion became perfectly constant, remaining so indefinitely.

The iron salts formed could not move with facility from the exposed surfaces through the hardened gelatine, and were easily outlined from their brown colour, as the whole apparatus was placed in a strong light.

\section{B. In a Uniform Magnetic Field.}

The cell was next placed in the magnetic field, which was kept practically uniform (about $15,650 \mathrm{H}$ ) for three hours, and galvanometer-readings taken as before:

The electrodes were magnetized before being introduced into the solution, so as to protect the point from the beginning. In order to prevent the influence of the rise of temperature due to the heating of the field coils of the electromagnet, the whole cell was packed with cotton-wool between the poles. As Gross and Andrews observed, the temperature effect was small, the solution rising but $0^{\circ} .7 \mathrm{C}$. in half an hour.

The curve fig. 1 (II.) shows the results of these observations. It is seen that the original current was, as before, to the point electrode, and about the same in value. This reversed after forty-five minutes, and rapidly increased to approximately twice its original value at the end of one hour and twenty minutes, and, instead of again reversing, remained indefinitely with the point electrode as a zinc. The distribution of the iron salts in this case was quite unlike the former. Notwithstanding the gelatine, the powerful magnetization of the exposed point gradually drew the iron salts from the disk as fast as they were formed, and concentrated them symmetrically about the point, giving the solution in this region an almost black appearance.

After waiting a sufficient time to be assured that further presence of iron salts would not effect the permanency of the existing electromotive force, the magnetic field was gradually decreased without ever breaking circuit, by increasing the 

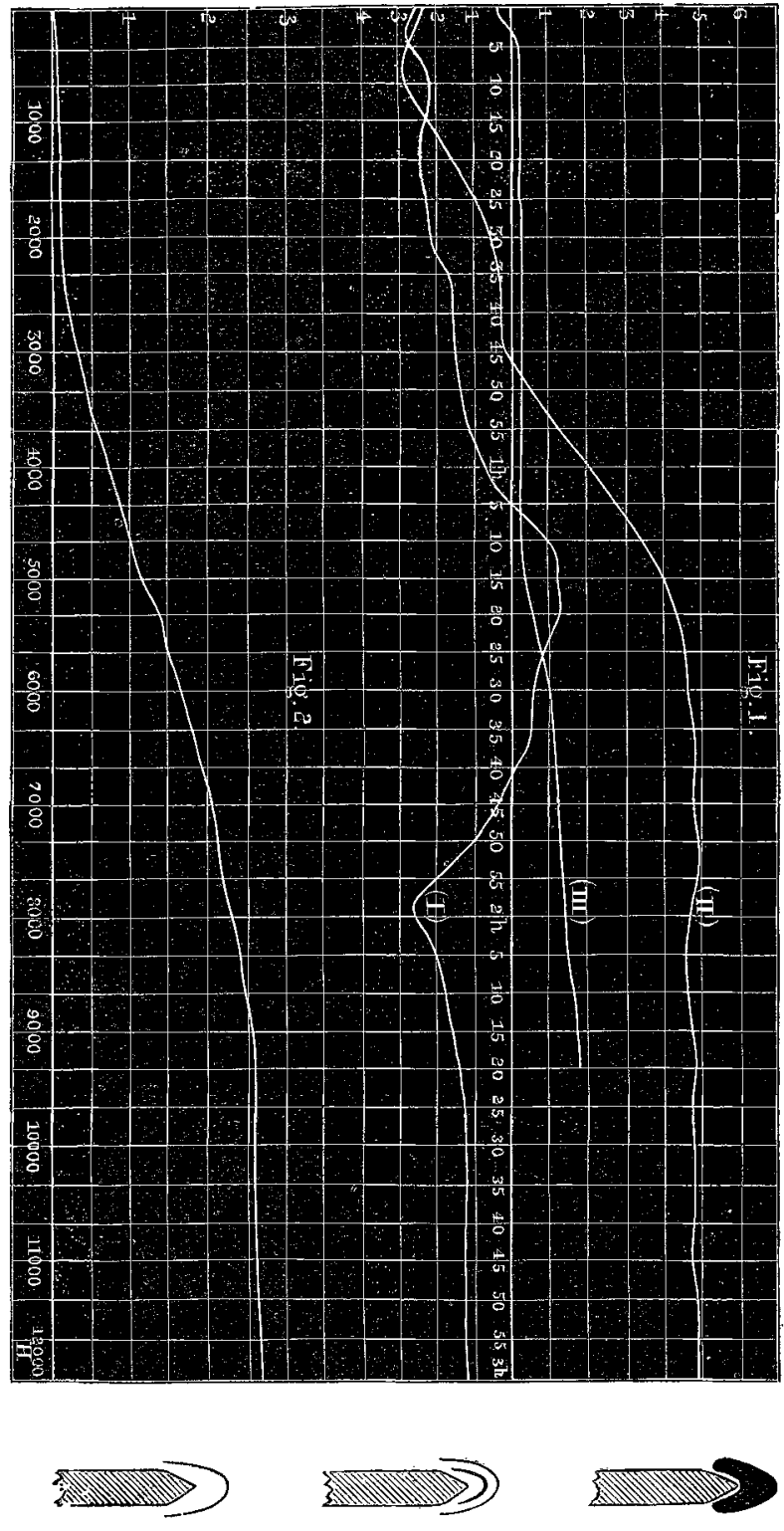

Fig. 3 
liquid resistance in the field current. This change of resistance was necessarily made more or less suddenly, and the deflexion experienced at each increase of resistance a not very sudden throw toward reversal, in every respect the same as had been repeatedly observed in the preliminary experiments, and very different from the characteristic "protective throw," which is always sudden and in one direction.

By simply varying the field current with care, as explained above, the deflexion could be reversed again and again at will, and could also be held at the zero of the scale, indicating no current at all, as long as desired. When once the field was entirely broken, the iron salts were released from the control of the exposed pole, seriously disturbed by gravity, and putting on the field again failed to reproduce the results noted above.

The only elements of difference in the two cases are, $(a)$ the magnetized condition of the metal, $(b)$ the distribution of the iron salts formed by the reaction.

Although, as the curves indicate, the average electromotive force with the magnetic field was much greater than in the former case, yet this electromotive force is due to the difference of action at the two exposed surfaces, and, as will be pointed out later, the total amount of iron dissolved and passing into solution in the two cases is probably not very different*. Quantitative experiments are wanting on this point.

The influence of the magnetized condition of the metal and its magnitude is exhibited in the phenomenon of the "protective throw," which is always observed with apparatus sufficiently delicate unless it is masked by other secondary phenomena.

Since the electrodes were embedded in hardened gelatine, there could be no convection-currents in the liquid, and this can be eliminated. Evidently the great difference in the behariour of the cell in the two experiments described is principally due, either directly or indirectly, to the distribution of the iron salts formed by the reaction in the two cases.

The principal time effects of the magnet were :-

(a) To produce a bigher potential at the point of greater magnetization.

(b) To increase the rate of change of the potential between the electrodes and the absolnte value of this potential difference.

(c) It also appears from both curves that after a certain distribution of iron salts is reached, further presence of the same does not affect the permanency of the current established.

* Fossati, Bolletino dell'Elettricista, 1890. 
Since the time effects of the magnet were so marked, it was thought possible that a "cumulative" effect, due to the earth's field alone, might be detected after a sufficient time had elapsed. The apparatus was made as delicate as possible, and parallel experiments conducted, the electrodes first being placed in the magnetic meridian, and afterwards perpendicular thereto. No positive difference could be detected.

\section{Convection-Currents in the Liquid.}

As has already been stated, the reversal of the current which regularly followed the "protective throw" was found by Messrs. Rowland and Bell to wholly disappear when hardened acidulated gelatine was substituted for the dilute acid solution, so that when the magnet was put on a permanent deflexion of much less magnitude was obtained instead of a transitory throw. This indicated that currents in the liquid cannot be neglected, and their study was next undertaken. Since hardened gelatine completely prevented the reversal of the current, and with no gelatine it regularly appeared after a short time, a large number of experiments were made, in which the amount of gelatine was varied continuously between these limits. As expected, the effects also varied-the greater the fluidity of the solution, the more quickly the reversal occurred.

In the light of what was already known concerning the presence of iron salts, some of the experiments were continued over a considerable time, and in others iron salts were introduced artificially, to increase the effects. It was soon found that by starting with a fresh hardened gelatine, with which the "protective throw" was the only feature, and gradually increasing the fluidity of the solution and the amount of iron salts present, both effects were exbibited at the making of the field - first, the sudden throw of the needle always in the direction to protect the point, and immediately thereafter the comparatively slow "concentration throw" in the opposite direction. By making the conditions still more unfavourable for the "protective throw," it gradually diminished until entirely masked by the second effect, so that making the field produced a deflexion in the direction indicating a current from the point.

With the proper conditions, both of these effects could be studied with the greatest ease: first, one made prominent, then both equal, then the other made prominent at will. The "protective throw" could be traced until it became a mere 
stationary tremor of the needle at the instant of its starting on the "concentration throw." This latter, though called a "throw," can be made to vary from an extremely slow continuous movement of the galvanometer deflexion, as in experiment $\mathrm{B}$ already described, to a comparatively rapid deflexion at the instant of making the magnet.

By using simply a dilute nitric-acid solution with no gelatine, and inserting a thick piece of glass between the electrodes, the concentration effect was delayed enough to allow the "protective throw" to first appear, with considerable iron salts in the solution; and on making the field both effects were observed as described above.

It now appears that the reversal of the current, uniformly observed in the experiments of Messrs. Rowland and Bell, was but a form of the "concentration throw" mentioned above, and that we can regard the substitution of the hardened acidulated gelatine for the dilute acid as merely separating these effects, so that the former can be studied by itself; in other words, the reversal of the current would have oceurred iust the same after a sufficient time had elapsed.

Turning to the experiments of Drs. Gross and Andrews, they employed but one magnetized electrode, which was not pointed. In this case the nature of the magnetic field at the two exposed surfaces would be very much more nearly the same than when a pointed electrode is employed. This arrangement is not, therefore, suited to bring out the delicate "protective throw," and it is not surprising that the concentration effect was the prominent feature observed.

We have now a complete reconciliation of the directly opposite results referred to in the introduction. The "protective throw" is due to the actual attraction of the magnet for the iron, and is always in the direction to protect the more strongly magnetized parts ; while the "concentration throw" is always in the opposite direction, and depends upon the distribution of the iron salts present in the solution, and the convection-currents in the liquid. The concentration of the products of the reaction about the point would tend to produce a ferrous reaction instead of a ferric, and experiment shows that a higher electromotive force is obtained with cells in which a ferrous reaction takes place than with those in which a ferric reaction occurs; and this change in the character of the reaction produced by the concentration probably accounts, at least in part, for the increased electromotive force at the point. 


\section{The Iron Salts about the Point Electrode.}

The effect of artificially stirring the liquid, and the direct influence of the fluid condition of the solution on the deflexions observed, at once suggested movements of the liquid, produced indirectly by the magnet. In order to locate these currents and determine their potence, a small cell was made of two rectangular pieces of glass held by stout rubber hands to thick rubber sides. Perforations in the sides admitted the electrodes, which were point and disk as before. The cell, between the poles of the electromagnet, was in a strong light, and the movements in the liquid were easily perceptible from the displacements of suspended particles introduced for the purpose. When very dilute nitric acid was placed in the cell and the magnet excited, some interesting phenomena were observed.

The liquid, at first colourless, almost immediately assumed a pale brown colour about the point, but nothing appeared at the disk electrode. The iron salts were drawn as soon ats formed towards the point electrode, since here the rate of variation of the square of the magnetic force is a maximum.

As more iron was dissolved, a surface approximating to an equipotential surface of the pointed pole, and enveloping the coloured iron salts, was observed enclosing the point and at some distance from it. The outline of the surface became darker in a short time, and finally two or more dark contours, separated by lighter portions and symmetrical with the outer one, appeared between it and the point, indicating maxima and minima of density. When the magnetic field was gradually increased, this surface usually enlarged without breaking up and holding the iron salts within it. On further strengthening the magnetic field to about $16,000 \mathrm{H}$, the ridges merged into one thick black envelope around the point.

This phenomenon is best studied with but little iron salts present, and by watching the point electrode with a microscope while the strength of the magnetic field is increased and decreased continuously. The sections (fig. 3) show the general form of these contours with different strengths of field.

Upon breaking the field everything dropped from the point suddenly to the bottom of the cell, and on making the field again it required a few seconds for the salts to reappear at the point.

This, at least partially, accounted for the sudden effects often noticed at breaking the field circuit, and the compara- 
tively small ones at "make," especially with certain saltsolutions, such as copper sulphate.

The outer envelope which held the iron salts together, and limited the immediate influence of the magnetized point, was distinctly defined within the liquid, and easily observed by the reflexion of the light from its convex surface.

The persistency with which the iron salts were held about the point was shown by moving the cell with respect to the electrodes, when the contour remained approximately intact, passing bodily through the liquid without being broken up.

\section{E. Electromagnetic Rotations.}

The small dust particles present in the liquid were drawn radially toward the point until they reached the surface described, when they pierced it and began to revolve rapidly about the point inside this surface, in the opposite direction to the currents of Ampere. Reversing the poles of the magnet produced surfaces of the same appearance but opposite rotations.

When the current from a Daniell cell was sent through it seemed to have very little effect upon the rotations, showing them to be controlled by the powerfully magnetized point.

The electromagnet was arranged with its field vertical, and the point electrode along the lines of force as before. This arrangement gave better control of the surfaces formed, since gravity now acted symmetrically about the point.

When a single iron rod about 3 millim. in diameter, and placed vertically in the eell, was substituted for the two electrodes, two rotations were observed which were uniformly dextro about the north-seeking pole of the rod, and lrvo about the south-seeking pole. About the central neutral portion no rotations were observed. When the rod was covered with a thin coating of vaseline the rotations entirely disappeared as expected. Wartmann* observed similar rotations about soft-iron cylinders adhering to the poles of a magnet, and he ascribed them to electric currents in the liquid which proceed from the periphery of the cell radially to the surface of the rod.

The explanation of these rotations follows at once from what we know of the time-effects produced by the magnet. A higher potential is always produced at points of greater magnetization, causing electric currents in the liquid from the more strongly magnetized to the weaker parts of the iron.

Applying this fact to the exposed conical point electrode, we see that local electric currents exist from its vertex to the other parts of the surface, returning by way of the metal. In the case of the vertical rod, these currents pass from the poles

* Philosophical Magazine, xxx. p. 268 (1847). 
at its ends, through the liquid, to the neutral portions, returning as before.

These currents*, under the influence of the poles themselves, would cause electromagnetic rotations of the liquid, as we find them. The mere mechanical influence of these rotations, as in the case when the liquid is artificially stirred, is to increase the chemical action upon the point, causing it to tend to act more like a zine, which experiment confirms.

F. Acids which attack Iron with the Evolution of Hydrogen.

Professor Rowland bad observed the "protective throw" with such acids to be extremely small, and difficult to detect except by very sensitive apparatus. The sensitive galvanometer was set up and every precution taken against inductive effects. A telescope and scale were used in this part of the work.

Several substances were first examined, among them being hydrochloric acid, acetic acid, perchloric acid, chlorine water, copper sulphate, ferric chloride, sulphuric acid, \&c., but as these observations added nothing to the results already obtained they are not given here.

After several trials a standard sulphuric-acid solution was made up as followe :-

Distilled water ..................... 10 grammes.

Gelatine ............................ 1 gramme.

C.P. Sulphuric acid, sp. gr. $1.826 \quad \ldots \quad 1.062$ gramme.

More strongly acidulated gelatine would not harden, and weaker solutions gave too small effects.

The "protective throw" was detected, but the point very soon became completely covered with minute bubbles of hydrogen, so that the electrodes had to be cleaned constantly.

The effect of adding hydrogen dioxide to the solution was next tried, since this would facilitate the removal of the hydrogen as soon as formed $\dagger$, which was thought to act merely mechauically.

When about 1 cubic centim. of $\mathrm{H}_{2} \mathrm{O}_{2}$ was added to the solution the "protective throw" became much more prominent, and the gas bubbles only appeared in small quantities after a considerable time. Further addition of small quantitics

* The rotations produced in liquids by axial currents, e.g. currents coinciding with the direction of the magnetic lines of force as distinct from radial currents, have been studied by Dr. Gore (Proceedings of the Royal Society, xxxiii. p. 151).

$\dagger$ J. M. Weeren, Berichte der Deutschen Chemischen Gesellschaft, No. 11 (1891). 
of the dioxide showed the "protective throw" to be very decided with sulphuric acid when the hydrogen is removed from the surface of the electrodes in this manner.

\section{G. The Electromotive Force.}

Several attempts were made to obtain the relation between the strength of field and the electromotive force developed in the "protective throw"; but it was difficult to obtain consistent readings owing to the trouble of balancing the original deflexion, and the small absolute values of this electromotive force when hardened gelatine was employed.

A curve was constructed, however, showing the variation of the galvanometer deflexion with the strength of field, using nitric-acid solution without gelatine. This is shown in fig. 2 .

The readings were taken one after another as rapidly as possible, to eliminate the damping effects of the iron salts formed.

The curve exhibits the general character of the variation. In the region from about $3500 \mathrm{H}$ to $8000 \mathrm{H}$ the greatest rate of change occurred, and beyond $10,000 \mathrm{H}$ the curve became nearly horizontal for the particular electrodes used. Curves were also constructed for the "concentration throw" on making the field under different conditions, and they were approximately right lines, more or less inclined according to the amount of iron salts present.

With the sulphuric-acid solution already given the electromotive force varied from 0.0033 to 0.0078 of a volt, while with the nitric-acid solution it became as great as 0.036 of a volt. In making all the solutions used with the different substances amounts were taken proportional to their particular molecular weights, and then halved or doubled until of suitable strength to give results with the galvanometer. It was thought possible at the beginning that this might lead to some relations between the protective results and the strengths of the particular solutions; but the general irregular character of the whole phenomenon prevented comparisons in this respect, and all that can be stated is, that both the "protective throw" and the concentration effect in general increased rapidly with the strength of the solution.

\section{H. Influence of a Periodic Magnetic Field upon the Cell.}

An experiment was made to determine the behaviour of the standard nitric-acid cell when the magnetic field was made and broken at regular intervals over a considerable time, and curves were drawn showing the variation of the "throw" with 
time, and the fluctuation of the original deflexion caused by this treatment. The strength of field was about $11,000 \mathrm{H}$., and the experiment was conducted without compensating the original deflexion, and by making the field for one minute, then breaking for one minute, and so on.

One of the curves is shown in fig. 1 (III.), in which positive ordinates are values of the concentration throw at " make," and negative ordinates the values of the "protective throw."

Experimenting was not begun until the gelatine had completely hardened, and since the electrodes would tend to become polarized while the gelatine was hardening, the "protective throw" was very small, and soon masked by the concentration effects.

After about five minutes, making the field had very little effect at all, but began to show decided "concentration throws" ten minutes later, and these rapidly increased with time, as the curve indicates.

Considering the fluctuation of the original deflexion, the effect of this periodic field was to tend to reverse it, just as in the case of the uniform field in experiment $B$, but much more slowly, since the field was on but half the time in this case.

The cell also showed the iron salts almost entirely about the point, forming a thick black envelope.

\section{Summary.}

The principal results of this investigation may be summarized as follows:-

Whenever iron is exposed to chemical action in a magnetic field, there are two directly opposite influences exerted.

(a) The direct influence of the magnetized condition of the metal, causing the more strongly magnetized parts to be protected from chemical action.

This is exhibited in the phenomenon of the "protective throw," which is always in the direction to protect the more strongly magnetized parts of magnetic electrodes.

The "protective throw" is small, often requiring delicate apparatus to detect it, and is soon masked by the secondary concentration effects.

As to the absence of the "protective throw" with acids which attack iron with the evolution of hydrogen, the hydrogen acts merely mechanically, and when removed by adding to the solution small quantities of hydrogen dioxide, the "protective throw" becomes very decided.

In the curve, fig. 2, representing the variations of the "protective throw" with the strength of the magnetic field, 
we trace at once the magnetization of the point-electrode. Since only the minute point was exposed to the liquid, it would become saturated for comparatively small magnetizing forces, and the curve indicates that this occurred at about $10,000 \mathrm{H}$., beyond which the curve becomes practically horizontal. This further establishes the direct connexion between this "throw" and the variation of the magnetization of the exposed point, and confirms the explanation of Professor Rowland, that it is due to the actual attraction of the magnet for the iron, and not to any molecular change produced by magnetization.

(b) The indirect influence of the magnet caused by the concentration of the products of the reaction about the more strongly magnetized parts of the iron.

This tends to produce a higher potential at the more strongly magnetized parts, and finally establishes permanent electric currents, which go in the liquid from the more strongly magnetized to the neutral parts of the iron.

This concentration-effect increases rapidly with the amount of iron salts present and the fluidity of the solution.

The convection-currents in the liquid are themselves a consequence of this same concentration, being electromagnetic rotations produced by the action of the magnet upon the local electric currents between different parts of the iron.

As to the permanent current due to the magnet which is finally set up between the electrodes, as shown in tig. 1 (II.), it is probably owing to a change in the character of the reaction produced by the concentration of the iron salts about the more strongly magnetized parts, which would tend to cause a ferrous instead of a ferric reaction to take place, and thus increase the electromotive force.

Physical Laboratory, Johns Hopkins University, May 1892.

Note.-Since the completion of the above investigation, a number of experiments have been performed similar to those of Professor Remsen. Starting with the known existence and direction of the electric currents in the liquid, it was thought that these might lead to some explanation of the . peculiar form of deposit in equipotential lines. A number of interesting facts have been noted, but they are withheld for further experiments. $G$. O. S.

Phil. Mag. S. 5. Vol. 35. No. 217. June 1893. 2 M 